PROCEEDINGS OF THE

AMERICAN MATHEMATICAL SOCIETY

Volume 137, Number 6, June 2009, Pages 1961-1969

S 0002-9939(08)09741-4

Article electronically published on December 30, 2008

\title{
AVERAGE BEHAVIOR OF FOURIER COEFFICIENTS OF CUSP FORMS
}

\author{
GUANGSHI LÜ
}

(Communicated by Ken Ono)

\begin{abstract}
Let $a_{0}(n)$ and $b_{0}(n)$ be the normalized Fourier coefficients of the two holomorphic Hecke eigenforms $f(z) \in S_{2 k}(\Gamma)$ and $\varphi(z) \in S_{2 l}(\Gamma)$ respectively. In 1999, Fomenko studied the following average sums of $a_{0}(n)$ and $b_{0}(n)$ :$$
\sum_{n \leq x} a_{0}(n)^{3}, \quad \sum_{n \leq x} a_{0}(n)^{2} b_{0}(n), \quad \sum_{n \leq x} a_{0}(n)^{2} b_{0}(n)^{2}, \quad \sum_{n \leq x} a_{0}(n)^{4} .
$$

In this paper, we are able to improve on Fomenko's results.

\section{INTRODUCTION AND MAIN RESULTS}

Let $S_{2 k}(\Gamma)$ be the space of holomorphic cusp forms of even weight $2 k$ for the full modular group $\Gamma=\mathrm{SL}(2, \mathbb{Z})$. Suppose that $f(z)$ and $\varphi(z)$ are two eigenfunctions of Hecke operators belonging to $S_{2 k}(\Gamma)$ and $S_{2 l}(\Gamma)$ respectively. Then the Hecke eigenforms $f(z)$ and $\varphi(z)$ have the following Fourier expansions at cusp $\infty$ :

$$
f(z)=\sum_{n=1}^{\infty} a(n) e^{2 \pi i n z}, \quad \varphi(z)=\sum_{n=1}^{\infty} b(n) e^{2 \pi i n z},
$$

where we normalize $f(z)$ and $\varphi(z)$ so that $a(1)=b(1)=1$. The Fourier coefficients of cusp forms are interesting objects (see [3, [12]). Instead of $a(n)$ and $b(n)$, one often considers the normalized Fourier coefficients

$$
a_{0}(n)=\frac{a(n)}{n^{k-\frac{1}{2}}}, \quad b_{0}(n)=\frac{b(n)}{n^{l-\frac{1}{2}}} .
$$

Hecke's theory (see for example [9] succeeds in developing the nice properties of the $L$-functions attached to cusp forms $f(z)$ and $\varphi(z)$, which are defined by

$$
\begin{aligned}
& L_{f}(s)=\sum_{n=1}^{\infty} a_{0}(n) n^{-s}=\prod_{p}\left\{\left(1-\alpha_{p} p^{-s}\right)\left(1-\bar{\alpha}_{p} p^{-s}\right)\right\}^{-1},\left|\alpha_{p}\right|=1, \alpha_{p}+\bar{\alpha}_{p}=a_{0}(p) ; \\
& L_{\varphi}(s)=\sum_{n=1}^{\infty} b_{0}(n) n^{-s}=\prod_{p}\left\{\left(1-\beta_{p} p^{-s}\right)\left(1-\bar{\beta}_{p} p^{-s}\right)\right\}^{-1},\left|\beta_{p}\right|=1, \beta_{p}+\bar{\beta}_{p}=b_{0}(p) .
\end{aligned}
$$

Received by the editors May 30, 2008, and, in revised form, August 28, 2008.

2000 Mathematics Subject Classification. Primary 11F30, 11F11, 11F66.

Key words and phrases. Fourier coefficients of cusp forms, Gelbart-Jacquet lift, $L$-function.

This work was supported by the National Natural Science Foundation of China (Grant No. 10701048).

(C)2008 American Mathematical Society Reverts to public domain 28 years from publication 
These Hecke $L$-functions are initially defined in the half-plane $\operatorname{Re}(s)>1$, and are then extended to entire functions satisfying functional equations of Riemann type (see for example 6], 9], 10]). Nowadays Hecke $L$-functions and their generalizations, the standard $L$-functions attached to cuspidal automorphic representations of $G L\left(n, \mathbb{A}_{\mathbb{Q}}\right)$, have become important tools in number theory under the guidance of the far-reaching Langlands program.

In 1999, Fomenko 4 studied the properties of some $L$-functions attached to several Hecke eigenforms, and proved the following results.

Theorem 0.1. For $x \rightarrow \infty$, we have

$$
\sum_{n \leq x} a_{0}(n)^{3} \ll x^{\frac{5}{6}+\varepsilon} .
$$

Theorem 0.2. For $x \rightarrow \infty$, we have

$$
\sum_{n \leq x} a_{0}(n)^{2} b_{0}(n) \ll x^{\frac{5}{6}+\varepsilon} .
$$

Theorem 0.3. Let $F_{1}$ be the Gelbart-Jacquet lift (see Gelbart and Jacquet [5]) on $G L(3)$ associated to $f$, and let $F_{2}$ be the Gelbart-Jacquet lift on GL(3) associated to $\varphi$. If $F_{1}$ and $F_{2}$ are distinct, then for $x \rightarrow \infty$, we have

$$
\sum_{n \leq x} a_{0}(n)^{2} b_{0}(n)^{2}=C x+O\left(x^{\frac{9}{10}+\varepsilon}\right),
$$

where $C>0$.

Theorem 0.4. For $x \rightarrow \infty$, we have

$$
\sum_{n \leq x} a_{0}(n)^{4}=C_{1} x \log x+C_{2} x+O\left(x^{\frac{9}{10}+\varepsilon}\right),
$$

where $C_{1}>0$.

In this paper we are able to prove the following results.

Theorem 1.1. For $x \rightarrow \infty$, we have

$$
\sum_{n \leq x} a_{0}(n)^{3} \ll x^{\frac{3}{4}+\varepsilon} .
$$

Theorem 1.2. For $x \rightarrow \infty$, we have

$$
\sum_{n \leq x} a_{0}(n)^{2} b_{0}(n) \ll x^{\frac{3}{4}+\varepsilon} .
$$

Theorem 1.3. If $f$ and $\varphi$ are distinct, then for $x \rightarrow \infty$, we have

$$
\sum_{n \leq x} a_{0}(n)^{2} b_{0}(n)^{2}=C x+O\left(x^{\frac{7}{8}+\varepsilon}\right),
$$

where $C>0$.

Theorem 1.4. For $x \rightarrow \infty$, we have

$$
\sum_{n \leq x} a_{0}(n)^{4}=C_{1} x \log x+C_{2} x+O\left(x^{\frac{7}{8}+\varepsilon}\right),
$$

where $C_{1}>0$. 


\section{Proof of Theorems 1.1 And 1.2}

The $L$-function attached to cusp forms $f, f$ and $\varphi$ is defined, for $\operatorname{Re}(s)>1$, by

$$
\begin{aligned}
& L_{f, f, \varphi}(s)=\prod_{p}\left\{\left(1-\alpha_{p}^{2} \beta_{p} p^{-s}\right)\left(1-\alpha_{p}^{2} \bar{\beta}_{p} p^{-s}\right)\left(1-\bar{\alpha}_{p}^{2} \beta_{p} p^{-s}\right)\left(1-\bar{\alpha}_{p}^{2} \bar{\beta}_{p} p^{-s}\right)\right\}^{-1} \\
& \times \prod_{p}\left\{\left(1-\beta_{p} p^{-s}\right)\left(1-\bar{\beta}_{p} p^{-s}\right)\right\}^{-2}=L_{\varphi}(s)^{2} L_{3}(s),
\end{aligned}
$$

where $L_{3}(s)$ is given by

$$
L_{3}(s)=\prod_{p}\left\{\left(1-\alpha_{p}^{2} \beta_{p} p^{-s}\right)\left(1-\alpha_{p}^{2} \bar{\beta}_{p} p^{-s}\right)\left(1-\bar{\alpha}_{p}^{2} \beta_{p} p^{-s}\right)\left(1-\bar{\alpha}_{p}^{2} \bar{\beta}_{p} p^{-s}\right)\right\}^{-1} .
$$

It was shown in 4 that for $\operatorname{Re}(s)>1$,

$$
\sum_{n=1}^{\infty} \frac{a_{0}(n)^{2} b_{0}(n)}{n^{s}}=L_{\varphi}(s)^{2} L_{3}(s) \prod_{p} M_{p}\left(p^{-s}\right)=L_{f, f, \varphi}(s) \prod_{p} M_{p}\left(p^{-s}\right),
$$

where

$$
M_{p}\left(p^{-s}\right)=1+\frac{3-2 a_{0}(p)^{2}-b_{0}(p)^{2}}{p^{2 s}}+\cdots+\frac{1}{p^{6 s}} .
$$

Obviously $\prod_{p} M_{p}\left(p^{-s}\right)$ converges uniformly in the half-plane $\operatorname{Re}(s) \geq \frac{1}{2}+\varepsilon$ for any $\varepsilon>0$.

It is easy to show that $L_{\varphi}(s) L_{3}(s)=L(F \times \varphi, s)$, where $F$ is the Gelbart-Jacquet lift on GL(3) associated to $f$. Therefore Shahidi's work [13] shows that $L_{f, f, \varphi}(s)$ is holomorphic on the whole complex plane and satisfies a functional equation of Riemann type.

In the special case of $f=\varphi$, we have that for $\operatorname{Re}(s)>1$,

$$
\sum_{n=1}^{\infty} \frac{a_{0}(n)^{3}}{n^{s}}=L_{f}(s)^{2} L_{3}(s) \prod_{p} M_{p}\left(p^{-s}\right)=L_{f, f, f}(s) \prod_{p} M_{p}\left(p^{-s}\right) .
$$

It follows from Shahidi's work [13 that $L_{f}(s)^{2} L_{3}(s)=L_{f, f, f}(s)$ is holomorphic on the whole plane and satisfies a functional equation of Riemann type.

We shall use the above properties to prove Theorems 1.1 and 1.2. Since the proofs of Theorems 1.1 and 1.2 are the same in essence, we shall give only the proof of Theorem 1.1 as an example.

To begin with, we recall two folklore results (see [2] and [10]).

Lemma 2.1. Let $L(f, s)$ be a Dirichlet series with Euler product of degree $m \geq 2$, which means that

$$
L(f, s)=\sum_{n=1}^{\infty} l_{f}(n) n^{-s}=\prod_{p<\infty} \prod_{j=1}^{m}\left(1-\frac{\alpha_{f}(p, j)}{p^{s}}\right)^{-1},
$$

where $\alpha_{f}(p, j), j=1, \cdots, m$, are the local parameters of $L(f, s)$ at prime $p$ and $l_{f}(n) \ll n^{\varepsilon}$. Assume that this series and its Euler product are absolutely convergent for $\operatorname{Re}(s)>1$. Assume also that it admits a meromorphic continuation to the whole 
complex plane $\mathbb{C}$ and satisfies a functional equation of Riemann type. Then we have that for $T \geq 1$,

$$
\int_{T}^{2 T}|L(f, 1 / 2+\varepsilon+i t)|^{2} d t \ll T^{\frac{m}{2}+\varepsilon} .
$$

Lemma 2.2. Let $L(f, s)$ be the same as in Lemma 2.1. Then for $0 \leq \sigma \leq 1$, we have

$$
L(f, \sigma+i t) \ll(|t|+1)^{\frac{m}{2}(1-\sigma)+\varepsilon} .
$$

It should be remarked that in Lemmas 2.1 and 2.2 we consider only the $t$-aspect in the analytic conductor introduced by Iwaniec and Kowalski [10. Therefore, like Fomenko's results, all constants in this paper depend on the weights of the corresponding cusp forms.

Proof of Theorem 1.1. By (2.2) and Perron's formula (see Proposition 5.54 in [10]), we have

$$
\sum_{n \leq x} a_{0}(n)^{3}=\frac{1}{2 \pi i} \int_{b-i T}^{b+i T} L_{f, f, f}(s) \prod_{p} M_{p}\left(p^{-s}\right) \frac{x^{s}}{s} d s+O\left(\frac{x^{1+\varepsilon}}{T}\right),
$$

where $b=1+\varepsilon$ and $1 \leq T \leq x$ is a parameter to be chosen later. Here we have used the Ramanujan-Petersson conjecture that $\left|a_{0}(n)\right| \leq d(n)=\sum_{d \mid n} 1$. This famous result was proved by Deligne 3 .

Next we move the integration to the parallel segment with $\operatorname{Re}(s)=\frac{1}{2}+\varepsilon$. By Cauchy's theorem, we have

$$
\begin{aligned}
\sum_{n \leq x} a_{0}(n)^{3}= & \frac{1}{2 \pi i}\left\{\int_{\frac{1}{2}+\varepsilon-i T}^{\frac{1}{2}+\varepsilon+i T}+\int_{\frac{1}{2}+\varepsilon+i T}^{b+i T}+\int_{b-i T}^{\frac{1}{2}+\varepsilon-i T}\right\} L_{f, f, f}(s) \prod_{p} M_{p}\left(p^{-s}\right) \frac{x^{s}}{s} d s \\
& +O\left(\frac{x^{1+\varepsilon}}{T}\right) \\
=: & I_{1}+I_{2}+I_{3}+O\left(\frac{x^{1+\varepsilon}}{T}\right) .
\end{aligned}
$$

To proceed further, we recall that $L_{f, f, f}(s)=L_{f}(s)^{2} L_{3}(s)$ is a Riemann-type nice $L$-function with Euler product of degree $m=8$.

For $I_{1}$, by (2.1) and Lemma 2.2 we have

$$
\begin{aligned}
I_{1} & \ll x^{\frac{1}{2}+\varepsilon}+x^{\frac{1}{2}+\varepsilon} \int_{1}^{T}\left|L_{f, f, f}(1 / 2+\varepsilon+i t) \prod_{p} M_{p}\left(p^{-\frac{1}{2}-i t}\right)\right| t^{-1} d t \\
& \ll x^{\frac{1}{2}+\varepsilon}+x^{\frac{1}{2}+\varepsilon} \int_{1}^{T}\left|L_{f, f, f}(1 / 2+\varepsilon+i t)\right| t^{-1} d t \\
& \ll x^{\frac{1}{2}+\varepsilon}+x^{\frac{1}{2}+\varepsilon} \int_{1}^{T}\left|L_{f}(1 / 2+\varepsilon+i t)^{2} L_{3}(1 / 2+\varepsilon+i t)\right| t^{-1} d t .
\end{aligned}
$$


Then by Lemma 2.1, we have

$$
\begin{aligned}
& I_{1} \ll x^{\frac{1}{2}+\varepsilon} \log T \\
& \cdot \max _{T_{1} \leq T}\left\{\frac{1}{T_{1}}\left(\int_{T_{1} / 2}^{T_{1}}\left|L_{f}(1 / 2+\varepsilon+i t)\right|^{2} d t\right)^{\frac{1}{2}}\right. \\
&\left.\quad\left(\int_{T_{1} / 2}^{T_{1}}\left|L_{f}(1 / 2+\varepsilon+i t) L_{3}(1 / 2+\varepsilon+i t)\right|^{2} d t\right)^{\frac{1}{2}}\right\} \\
& \ll x^{\frac{1}{2}+\varepsilon} T^{1+\varepsilon}
\end{aligned}
$$

where we have used

$$
\int_{T_{1} / 2}^{T_{1}}\left|L_{f}(1 / 2+\varepsilon+i t)\right|^{2} d t \ll T_{1}^{1+\varepsilon}
$$

and

$$
\int_{T_{1} / 2}^{T_{1}}\left|L_{f}(1 / 2+\varepsilon+i t) L_{3}(1 / 2+\varepsilon+i t)\right|^{2} d t \ll T_{1}^{3+\varepsilon}
$$

For the integral over the horizontal segments, we use Lemma 2.2 with $m=8$ to get

$$
\begin{aligned}
I_{2}+I_{3} & \ll \int_{\frac{1}{2}+\varepsilon}^{b} x^{\sigma}\left|L_{f, f, f}(\sigma+i T)\right| T^{-1} d \sigma \\
& \ll \max _{\frac{1}{2}+\varepsilon \leq \sigma \leq b} x^{\sigma} T^{4(1-\sigma)+\varepsilon} T^{-1}=\max _{\frac{1}{2}+\varepsilon \leq \sigma \leq b}\left(\frac{x}{T^{4}}\right)^{\sigma} T^{3+\varepsilon} \\
& \ll \frac{x^{1+\varepsilon}}{T}+x^{\frac{1}{2}+\varepsilon} T^{1+\varepsilon} .
\end{aligned}
$$

From (2.4), (2.6) and (2.7), we have

$$
\sum_{n \leq x} a_{0}(n)^{3} \ll \frac{x^{1+\varepsilon}}{T}+x^{\frac{1}{2}+\varepsilon} T^{1+\varepsilon} .
$$

On taking $T=x^{\frac{1}{4}}$ in $(2.8)$, we have

$$
\sum_{n \leq x} a_{0}(n)^{3} \ll x^{\frac{3}{4}+\varepsilon}
$$

This completes the proof of Theorem 1.1.

\section{Proof of Theorems 1.3 and 1.4}

If $f(z) \neq \varphi(z)$, it was shown in [4] that for $\operatorname{Re}(s)>1$,

$$
\sum_{n=1}^{\infty} \frac{a_{0}(n)^{2} b_{0}(n)^{2}}{n^{s}}=\zeta(s) L\left(s, F_{1}\right) L\left(s, F_{2}\right) L\left(s, F_{1} \times F_{2}\right) \prod_{p} Q_{p}\left(p^{-s}\right),
$$

where

$$
Q_{p}\left(p^{-s}\right)=1-\frac{\gamma_{2}(p)}{p^{2 s}}+\cdots+\frac{1}{p^{14 s}} .
$$

Here $F_{1}$ is the Gelbart-Jacquet lift (see Gelbart and Jacquet [5]) on GL(3) associated to $f, F_{2}$ is the Gelbart-Jacquet lift on GL(3) associated to $\varphi$, and $F_{1} \times F_{2}$ is the 
Rankin-Selberg convolution of $F_{1}$ and $F_{2}$. The $L$-functions $L\left(s, F_{1}\right), L\left(s, F_{2}\right)$ and $L\left(s, F_{1} \times F_{2}\right)$ are defined by

$$
\begin{gathered}
L\left(s, F_{1}\right)=\prod_{p}\left(1-\frac{\alpha_{p}^{2}}{p^{s}}\right)^{-1}\left(1-\frac{1}{p^{s}}\right)^{-1}\left(1-\frac{\bar{\alpha}_{p}^{2}}{p^{s}}\right)^{-1}, \\
L\left(s, F_{2}\right)=\prod_{p}\left(1-\frac{\beta_{p}^{2}}{p^{s}}\right)^{-1}\left(1-\frac{1}{p^{s}}\right)^{-1}\left(1-\frac{\bar{\beta}_{p}^{2}}{p^{s}}\right)^{-1}, \\
L\left(s, F_{1} \times F_{2}\right)=\prod_{p}\left(1-\frac{\alpha_{p}^{2} \beta_{p}^{2}}{p^{s}}\right)^{-1}\left(1-\frac{\alpha_{p}^{2}}{p^{s}}\right)^{-1}\left(1-\frac{\alpha_{p}^{2} \bar{\beta}_{p}^{2}}{p^{s}}\right)^{-1}\left(1-\frac{\beta_{p}^{2}}{p^{s}}\right)^{-1} \\
\times\left(1-\frac{1}{p^{s}}\right)^{-1}\left(1-\frac{\bar{\beta}_{p}^{2}}{p^{s}}\right)^{-1}\left(1-\frac{\bar{\alpha}_{p}^{2} \beta_{p}^{2}}{p^{s}}\right)^{-1}\left(1-\frac{\bar{\alpha}_{p}^{2}}{p^{s}}\right)^{-1}\left(1-\frac{\bar{\alpha}_{p}^{2} \bar{\beta}_{p}^{2}}{p^{s}}\right)^{-1} .
\end{gathered}
$$

In fact $L\left(s, F_{i}\right), i=1,2$, are entire functions and $L\left(1, F_{i}\right) \neq 0$. Furthermore, from the assumption that $f$ and $\varphi$ are distinct, one can easily show that $F_{1}$ and $F_{2}$ are distinct. Therefore $L\left(s, F_{1} \times F_{2}\right)$ is also an entire function and $L\left(1, F_{1} \times F_{2}\right) \neq 0$. All these $L$-functions satisfy functional equations of Riemann type (see [5], 6], [7], 8] and [10]). Therefore $\zeta(s) L\left(s, F_{1}\right) L\left(s, F_{2}\right) L\left(s, F_{1} \times F_{2}\right)$ only has a simple pole at the point $s=1$ and satisfies a functional equation of Riemann type. In addition the product $\prod_{p} Q_{p}\left(p^{-s}\right)$ converges uniformly for any $\varepsilon>0$ in the halfplane $\operatorname{Re}(s) \geq \frac{1}{2}+\varepsilon$.

If $f(z)=\varphi(z)$, it was shown by Moreno and Shahidi [1] that for $\operatorname{Re}(s)>1$,

$$
\sum_{n=1}^{\infty} \frac{a_{0}(n)^{4}}{n^{s}}=\zeta(s) L(s, F)^{2} L(s, F \times F) \prod_{p} T_{p}\left(p^{-s}\right),
$$

where

$$
T_{p}\left(p^{-s}\right)=1-\frac{\delta_{2}(p)}{p^{2 s}}+\cdots+\frac{1}{p^{14 s}} .
$$

Here $F$ is the Gelbart-Jacquet lift on GL(3) associated to $f$. By the above Euler product of $L\left(s, F_{1} \times F_{1}\right)$ with $F_{1}=F_{2}=F$, we have

$$
\zeta(s) L(s, F)^{2} L(s, F \times F)=\zeta(s) L(s, F)^{3} L\left(s, F, \vee^{2}\right),
$$

where

$$
\begin{aligned}
L\left(s, F, \vee^{2}, s\right)= & \prod_{p}\left(1-\frac{\alpha_{p}^{4}}{p^{s}}\right)^{-1}\left(1-\frac{\alpha_{p}^{2}}{p^{s}}\right)^{-1}\left(1-\frac{1}{p^{s}}\right)^{-1} \\
& \times\left(1-\frac{1}{p^{s}}\right)^{-1}\left(1-\frac{\bar{\alpha}_{p}^{2}}{p^{s}}\right)^{-1}\left(1-\frac{\bar{\alpha}_{p}^{4}}{p^{s}}\right)^{-1}
\end{aligned}
$$

is the symmetric square $L$-function of the form $F$. Bump and Ginzburg [1 proved that the function $L\left(s, F, \vee^{2}\right)=\zeta(s) L\left(\operatorname{Sym}^{4} f, s\right)$ has a simple pole at $s=1$ and is analytic everywhere else. Therefore $\zeta(s) L(s, F)^{2} L(s, F \times F)$ has a double pole at $s=1$ and is analytic at other points. The product $\prod_{p} T_{p}\left(p^{-s}\right)$ converges uniformly for any $\varepsilon>0$ in the half-plane $\operatorname{Re}(s) \geq \frac{1}{2}+\varepsilon$ and has no zeros in this region. 
Now we use these properties of $L$-functions to complete the proofs of Theorems 1.3 and 1.4. The proofs of Theorems 1.3 and 1.4 are very similar in essence; therefore we give the proof only of Theorem 1.4.

Proof of Theorem 1.4. By (3.1) and Perron's formula, we have

$$
\sum_{n \leq x} a_{0}(n)^{4}=\frac{1}{2 \pi i} \int_{b-i T}^{b+i T} \zeta(s) L(s, F)^{2} L(s, F \times F) \prod_{p} T_{p}\left(p^{-s}\right) \frac{x^{s}}{s} d s+O\left(\frac{x^{1+\varepsilon}}{T}\right),
$$

where $b=1+\varepsilon$ and $1 \leq T \leq x$ is a parameter to be chosen later.

Then we move the integration to the parallel segment with $\operatorname{Re}(s)=\frac{1}{2}+\varepsilon$. By Cauchy's theorem, we have

$$
\begin{aligned}
& \sum_{n \leq x} a_{0}(n)^{4} \\
& =\frac{1}{2 \pi i}\left\{\int_{\frac{1}{2}+\varepsilon-i T}^{\frac{1}{2}+\varepsilon+i T}+\int_{\frac{1}{2}+\varepsilon+i T}^{b+i T}+\int_{b-i T}^{\frac{1}{2}+\varepsilon-i T}\right\} \\
& \zeta(s) L(s, F)^{2} L(s, F \times F) \prod_{p} T_{p}\left(p^{-s}\right) \frac{x^{s}}{s} d s \\
& +\operatorname{Res}_{s=1}\left\{\zeta(s) L(s, F)^{2} L(s, F \times F) \prod_{p} T_{p}\left(p^{-s}\right) \frac{x^{s}}{s}\right\}+O\left(\frac{x^{1+\varepsilon}}{T}\right) \\
& =: C_{1} x \log x+C_{2} x+J_{1}+J_{2}+J_{3}+O\left(\frac{x^{1+\varepsilon}}{T}\right) .
\end{aligned}
$$

To go further, we recall that $\zeta(s) L(s, F)^{2} L(s, F \times F)$ is a Riemann-type nice $L$-function with Euler product of degree $m=16$.

For $J_{1}$, by (3.1) and Lemma 2.2 we have

$$
\begin{aligned}
J_{1} \ll & x^{\frac{1}{2}+\varepsilon} \int_{1}^{T}\left|L(1 / 2+\varepsilon+i t, F)^{2} L(1 / 2+\varepsilon+i t, F \times F) \zeta(1 / 2+\varepsilon+i t)\right| t^{-1} d t \\
& +x^{\frac{1}{2}+\varepsilon} .
\end{aligned}
$$

Then by Lemma 2.1, we have

$$
\begin{aligned}
J_{1} \ll & x^{1 / 2+\varepsilon} \log T \max _{T_{1} \leq T}\left\{\frac{1}{T_{1}}\left(\int_{T_{1} / 2}^{T_{1}}\left|L(1 / 2+\varepsilon+i t, F)^{2}\right|^{2} d t\right)^{\frac{1}{2}}\right. \\
& \left.\times\left(\int_{T_{1} / 2}^{T_{1}}|L(1 / 2+\varepsilon+i t, F \times F) \zeta(1 / 2+\varepsilon+i t)|^{2} d t\right)^{\frac{1}{2}}\right\} \\
\ll & x^{\frac{1}{2}+\varepsilon} T^{3+\varepsilon},
\end{aligned}
$$

where we have used

$$
\begin{gathered}
\int_{T_{1} / 2}^{T_{1}}\left|L(1 / 2+\varepsilon+i t, F)^{2}\right|^{2} d t \ll T^{3+\varepsilon} \\
\int_{T_{1} / 2}^{T_{1}}|L(1 / 2+\varepsilon+i t, F \times F) \zeta(1 / 2+\varepsilon+i t)|^{2} d t \ll T^{5+\varepsilon} .
\end{gathered}
$$


For the integral over the horizontal segments, we use Lemma 2.2 with $m=16$ to get

$$
\begin{aligned}
J_{2}+J_{3} & \ll \int_{\frac{1}{2}+\varepsilon}^{b} x^{\sigma}\left|\zeta(\sigma+i T) L(\sigma+i T, F)^{2} L(\sigma+i T, F \times F)\right| T^{-1} d \sigma \\
& \ll \max _{\frac{1}{2}+\varepsilon \leq \sigma \leq b} x^{\sigma} T^{8(1-\sigma)+\varepsilon} T^{-1}=\max _{\frac{1}{2}+\varepsilon \leq \sigma \leq b}\left(\frac{x}{T^{8}}\right)^{\sigma} T^{7+\varepsilon} \\
& \ll \frac{x^{1+\varepsilon}}{T}+x^{\frac{1}{2}+\varepsilon} T^{3+\varepsilon} .
\end{aligned}
$$

From (3.4), (3.5) and (3.6), we obtain

$$
\sum_{n \leq x} a_{0}(n)^{4}=C_{1} x \log x+C_{2} x+O\left(\frac{x^{1+\varepsilon}}{T}\right)+O\left(x^{\frac{1}{2}+\varepsilon} T^{3+\varepsilon}\right) .
$$

On taking $T=x^{\frac{1}{8}}$ in (3.7), we have

$$
\sum_{n \leq x} a_{0}(n)^{4}=C_{1} x \log x+C_{2} x+O\left(x^{\frac{7}{8}+\varepsilon}\right) .
$$

This completes the proof of Theorem 1.4.

\section{ACKNOWLEDGEMENTS}

This work was completed when the author visited Stanford University with support from the China Scholarship Council. The author would like to thank Professor K. Soundararajan and Professor Jianya Liu for their encouragement. The author is grateful to the referee for detailed suggestions and valuable comments.

\section{REFERENCES}

[1] D. Bump and D. Ginzburg, Symmetric square $L$-functions on $G L(r)$, Ann. of Math., 136(1992), 137-205. MR1173928 (93i:11058)

[2] K. Chandrasekharan and R. Narasimhan, Functional equations with multiple gamma factors and the average order of arithmetical functions, Ann. of Math., 76(1962), 93-136. MR0140491 (25:3911)

[3] P. Deligne, La Conjecture de Weil, Inst. Hautes Études Sci. Publ. Math., 43(1974), 273-307. MR0340258 (49:5013)

[4] O.M. Fomenko, Fourier coefficients of parabolic forms and automorphic $L$-functions, J. of Math. Sci., 95(1999), 2295-2316. MR1691291 (2001a:11077)

[5] S. Gelbart and H. Jacquet, A relation between automorphic representations of GL(2) and GL(3), Ann. Sci. École Norm. Sup., 11(1978), 471-552. MR.533066 (81e:10025)

[6] D. Goldfeld, Automorphic Forms and $L$-functions for the Group $G L(n, \mathbb{R})$, Cambridge Studies in Advanced Mathematics, 99, Cambridge University Press, 2006. MR2254662 (2008d:11046)

[7] H. Jacquet, I. Piatetski-Shapiro and J. Shalika, Rankin-Selberg convolutions, Amer. J. Math., 105(1983), 367-464. MR701565 (85g:11044)

[8] H. Jacquet and J. Shalika, Rankin-Selberg convolutions: Archimedean theory, Weizmann Inst. Sci., 1(1990), 125-208. MR1159102 (93d:22022)

[9] H. Iwaniec, Topics in Classical Automorphic Forms, Grad. Stud. Math., 17, Amer. Math. Soc., Providence, RI, 1997. MR1474964 (98e:11051)

[10] H. Iwaniec and E. Kowalski, Analytic Number Theory, Amer. Math. Soc. Colloquium Publ., 53, Amer. Math. Soc., Providence, RI, 2004. MR2061214 (2005h:11005)

[11] C.J. Moreno and F. Shahidi, The fourth moment of the Ramanujan $\tau$-function, Math. Ann., 266(1983), 431-446. MR724740(85i:11039)

[12] R.A. Rankin, Contributions to the theory of Ramanujan's function $\tau(n)$ and similar arithmetical functions, II. The order of the Fourier coefficients of the integral modular forms, Proc. Cambridge Phil. Soc., 35(1939), 357-372. 
[13] F. Shahidi, Third symmetric power $L$-functions for GL(2), Compos. Math., 70(1989), 245-273. MR:1002045 (90m:11081)

[14] G. Shimura, On the holomorphy of certain Dirichlet series, Proc. London Math. Soc., 31(1975), 79-98. MR0382176 (52:3064)

Department of Mathematics, Shandong University, Jinan, Shandong, 250100, People's Republic of ChinA

E-mail address: gslv@sdu.edu.cn 